\title{
Ambient particulate matter accelerates coagulation via an IL-6-dependent pathway
}

\author{
Gökhan M. Mutlu, ${ }^{1}$ David Green,, ${ }^{2}$ Amy Bellmeyer, ${ }^{1}$ Christina M. Baker, ${ }^{1}$ Zach Burgess, ${ }^{1}$ \\ Nalini Rajamannan, ${ }^{3}$ John W. Christman, ${ }^{4}$ Nancy Foiles, ${ }^{2}$ David W. Kamp, ${ }^{1}$ Andrew J. Ghio, ${ }^{5}$ \\ Navdeep S. Chandel, ${ }^{1}$ David A. Dean, ${ }^{1}$ Jacob I. Sznajder, ${ }^{1}$ and G.R. Scott Budinger ${ }^{1}$
}

\begin{abstract}
1Division of Pulmonary and Critical Care Medicine, ${ }^{2}$ Division of Hematology and Oncology, and ${ }^{3}$ Division of Cardiology, Northwestern University Feinberg School of Medicine, Chicago, Illinois, USA. ${ }^{4}$ Section of Pulmonary, Critical Care, and Sleep Medicine, University of Illinois, Chicago, Illinois, USA. 5United States Environmental Protection Agency, Research Triangle Park, North Carolina, USA.
\end{abstract}

\begin{abstract}
The mechanisms by which exposure to particulate matter increases the risk of cardiovascular events are not known. Recent human and animal data suggest that particulate matter may induce alterations in hemostatic factors. In this study we determined the mechanisms by which particulate matter might accelerate thrombosis. We found that mice treated with a dose of well characterized particulate matter of less than $10 \mu \mathrm{M}$ in diameter exhibited a shortened bleeding time, decreased prothrombin and partial thromboplastin times (decreased plasma clotting times), increased levels of fibrinogen, and increased activity of factor II, VIII, and X. This prothrombotic tendency was associated with increased generation of intravascular thrombin, an acceleration of arterial thrombosis, and an increase in bronchoalveolar fluid concentration of the prothrombotic cytokine IL-6. Knockout mice lacking IL-6 were protected against particulate matter-induced intravascular thrombin formation and the acceleration of arterial thrombosis. Depletion of macrophages by the intratracheal administration of liposomal clodronate attenuated particulate matter-induced IL-6 production and the resultant prothrombotic tendency. Our findings suggest that exposure to particulate matter triggers IL- 6 production by alveolar macrophages, resulting in reduced clotting times, intravascular thrombin formation, and accelerated arterial thrombosis. These results provide a potential mechanism linking ambient particulate matter exposure and thrombotic events.
\end{abstract}

\section{Introduction}

Epidemiologic studies have consistently linked morbidity and mortality from ischemic cardiovascular events, such as acute myocardial infarction (1-3), discharge of implanted automatic cardioverter defibrillators (4), hospitalizations for acute decompensation of patients with congestive heart failure (5), and ischemic stroke (6), with acute exposure to particulate matter (PM).

Exposure of rodents to PM has been reported to result in bronchoalveolar lavage (BAL) fluid pleocytosis, platelet function abnormalities, and hemostatic alterations that culminate in intravascular thrombosis $(7,8)$. The doses used in these models are similar, when adjusted for differences in tidal volume, to the doses to which humans might be exposed in urban centers in the United States. The mechanisms linking PM exposure to the development of thrombosis, however, have not been elucidated.

IL-6 is a cytokine that is released from the lung in response to a wide variety of inflammatory stimuli (9). The transcriptional targets of IL-6 include several proteins that either increase the transcription of procoagulant proteins or decrease the transcription of anticoagulant proteins (10). Increased levels of IL- 6 or its transcriptional target, C-reactive protein, have been consistently linked to ischemic cardiovascular events (11-14). Exposure of healthy individuals and patients with coronary artery disease to PM has been shown to increase the serum levels of IL-6 and C-reactive protein (9).

Nonstandard abbreviations used: ADP, adenosine diphosphate; aPTT, activated partial thromboplastin time; BAL, bronchoalveolar lavage; PM, particulate matter; $\mathrm{PM}_{10}$, PM of less than $10 \mu \mathrm{m}$ in diameter; PT, prothrombin time; TAT, thrombin-antithrombin.

Conflict of interest: The authors have declared that no conflict of interest exists. Citation for this article: J. Clin. Invest. 117:2952-2961 (2007). doi:10.1172/JCI30639.
While developing a model of PM exposure in mice (15), we clinically observed grossly reduced bleeding and accelerated blood clotting during thoracotomy in C57BL6/J mice 24 hours after the intratracheal administration of PM compared with PBS-treated control mice. Consistent with this observation, the bleeding time was shortened, prothrombin and partial thromboplastin times were reduced, and plasma factor VIII activity was increased in PM-treated compared with PBS-treated animals. This PM-induced prothrombotic tendency resulted in intravascular generation of thrombin and accelerated carotid artery thrombus formation after $\mathrm{FeCl}_{3}$-induced injury. Mice lacking IL-6 (IL6 ${ }^{-/-}$mice) or depleted of alveolar macrophages did not develop a prothrombotic tendency in response to PM. Our results provide a novel mechanistic link between exposure of the respiratory tract to PM and the development of thrombosis.

\section{Results}

Exposure to moderate doses of PM causes a prothrombotic tendency in mice. To study the effects of PM in cardiovascular disease, we established a mouse model of PM exposure, which consisted of intratracheal instillation of $10 \mu \mathrm{g}$ of PM less than $10 \mu \mathrm{m}$ in diameter $\left(\mathrm{PM}_{10}\right)$ in $50 \mu \mathrm{l}$ of sterile PBS. The PM used in our experiments was collected by baghouse from ambient air in Düsseldorf, Germany, and has been previously characterized (16). The dose of $\mathrm{PM}_{10}$ used in our experiments is equivalent (by weight) to the daily dose to which humans are exposed when the ambient PM level is approximately $150 \mu \mathrm{g} / \mathrm{m}^{3}$, which corresponds to a moderate elevation in PM levels (17).

During excision of the lungs of C57BL6/J $\left(I L 6^{+/+}\right)$mice treated with PM 24 hours earlier, we clinically observed grossly reduced bleeding and accelerated clot formation compared with PBS-treated animals. This observation led us to examine the coagulation profile of PM-treated mice (Figure 1). Compared with PBS-treated 

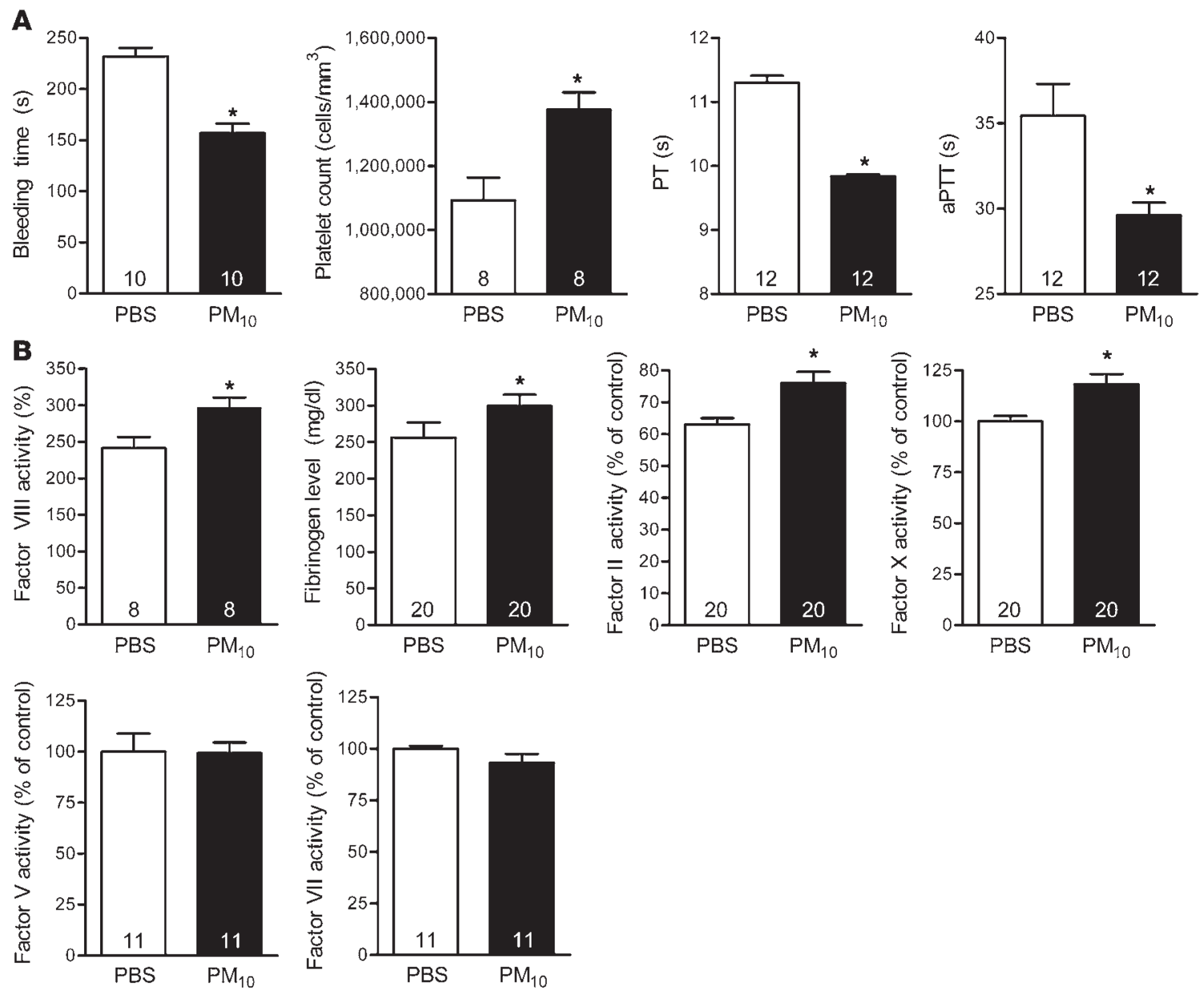

\section{Figure 1}

Alterations in hemostasis in wild-type mice treated with PM. Airborne $\mathrm{PM}_{10}(10 \mu \mathrm{g}$ in $50 \mu \mathrm{l}$ PBS) or PBS alone was intratracheally instilled into mouse lungs, and 24 hours later measurements of hemostasis were made. (A) Bleeding time, platelet count, PT, and aPTT measurements. (B) Fibrinogen and coagulation factors (factors II, V, VII, VIII, and X). The number inside each bar represents the number of animals for each group of experiments. ${ }^{*} P<0.05$ for comparison between PM- and PBS-treated mice, $n \geq 8$ in each treatment group.

mice, the bleeding time (tail vein cut) was reduced significantly in PM-exposed animals (232 \pm 26 versus $157 \pm 29$ seconds, respectively; $n=10 ; P<0.001)$. To study the effects of PM exposure on coagulation, blood was collected from mice by right atrial puncture 24 hours after $\mathrm{PM}_{10}$ or PBS instillation. Compared with PBS-treated mice, mice exposed to $\mathrm{PM}_{10}$ showed an increase in the platelet count $\left(1,376,000 \pm 154,000\right.$ versus $1,093,000 \pm 201,000$ cells $/ \mathrm{mm}^{3} ; n=8$; $P<0.05)$ and a reduction in the prothrombin time (PT) $(9.84 \pm 0.10$ versus $11.30 \pm 0.39$ seconds; $n=12 ; P<0.001)$ and the activated partial thromboplastin time (aPTT) $(29.62 \pm 1.85$ versus $35.44 \pm 5.92$ seconds; $n=12 ; P<0.05$ ). Consistent with a PM-induced prothrombotic tendency, the plasma level of fibrinogen and activities of factor II, VIII, and X significantly increased in PM-treated mice (Figure 1), while the activities of factor $V$ and factor VII were not changed. Our data showing that the intratracheal instillation of PM results in prothrombotic tendency are consistent with the observations of other investigators in human subjects $(18,19)$ and animals $(7,8,20)$.

Exposure to PM does not activate platelets. To determine the effect of PM on platelet function, we performed flow cytometry using CD62P antibody (Emfret Analytics) in the absence and presence of adenosine diphosphate (ADP) (21). Mice exposed to PM had simi- lar activation of platelets at baseline. Addition of ADP increased the percentage of activated platelets similarly in both groups of mice (Figure 2), suggesting that the reduction in bleeding time we observed in PM-treated animals was due to factors other than direct activation of the platelets.

Exposure to PM is sufficient to generate intravascular thrombin and to accelerate occlusion of the carotid artery after $\mathrm{FeCl}_{3}$-induced injury. To discover whether the prothrombotic tendency induced by PM was sufficient to cause the intravascular generation of thrombin, we measured the level of covalently linked thrombin-antithrombin (TAT) complexes in the plasma of mice 24 hours after the instillation of $\mathrm{PM}_{10}$. Plasma levels of TAT complexes were nearly 4 times higher in PM-exposed mice compared with controls $(16.4 \pm 2.9$ versus $5.1 \pm 1.0 \mathrm{ng} / \mathrm{ml}$; $n=8 ; P=0.002)$, suggesting that PM exposure induces intravascular thrombosis (Figure 3A). We next sought to determine whether exposure to PM could accelerate thrombus generation in a well-established model of carotid artery injury. We directly applied $10 \% \mathrm{FeCl}_{3}$ to the left carotid artery as previously described (22). The left carotid artery was then isolated, and the time to occlusion was measured as the disappearance of flow by 2D/Doppler ultrasonography (Figure 3B). Histologic examination was performed immediately after loss of 

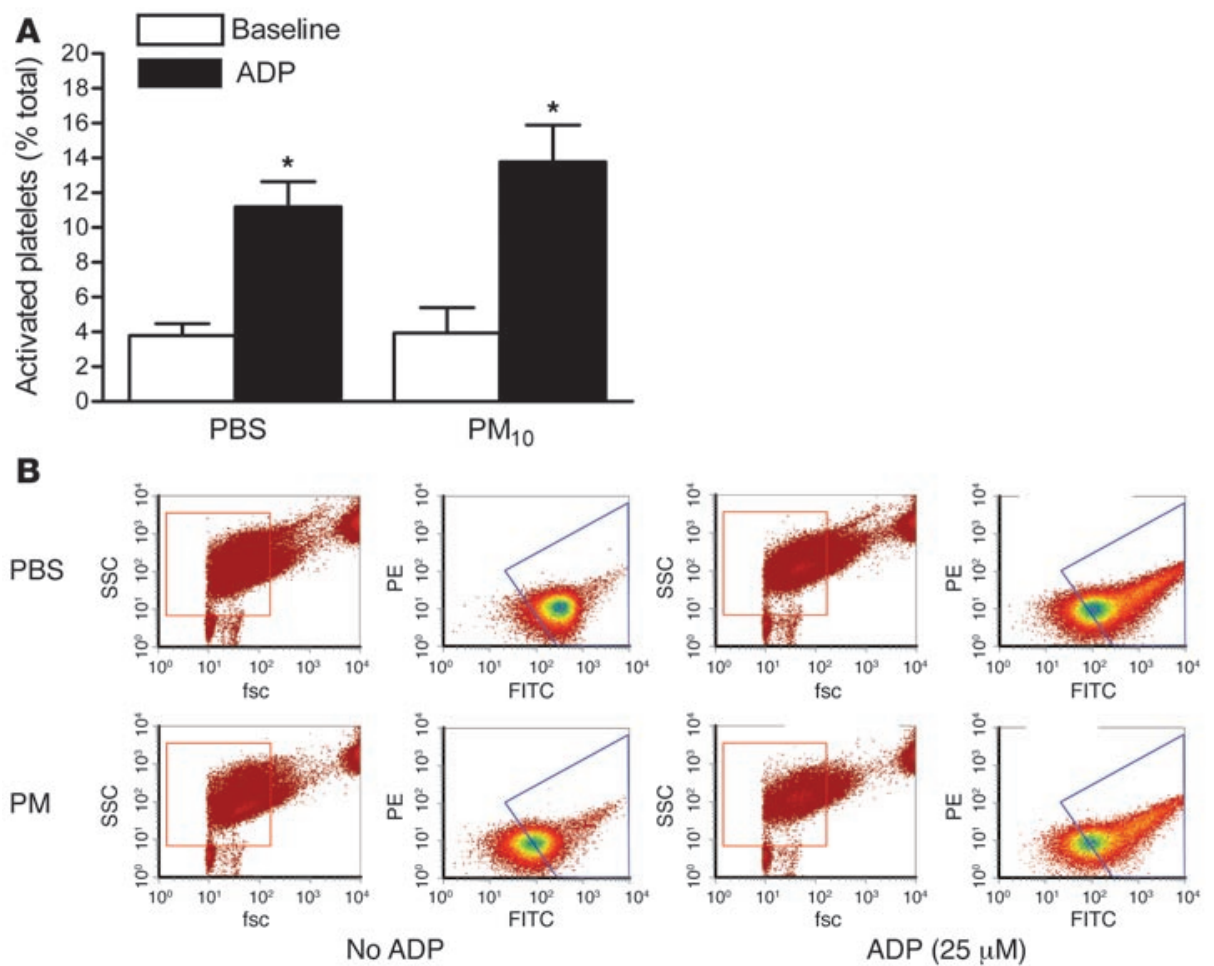

\section{Figure 2}

Activation of platelets in mice treated with PM. Airborne $\mathrm{PM}_{10}(10 \mu \mathrm{g}$ in $50 \mu \mathrm{l}$ PBS) or PBS alone was intratracheally instilled into mouse lungs, and 24 hours later platelet activation was measured via FACS analysis using CD62P antibody in the absence or presence of ADP. (A and $\mathbf{B}$ ) Percent activation of platelets from mice exposed to $\mathrm{PM}_{10}$ or PBS (A) in the absence or presence of ADP (B). ${ }^{*} P<0.05$ for comparison between $\mathrm{PM}$ and PBS-treated mice, $n \geq 5$ for each treatment group. fsc, forward scatter; ssc, side scatter. blood flow to confirm complete occlusion (Figure 3C). Mice treated with PM 24 hours previously had a significant reduction in the time to carotid occlusion (Figure 3D) when compared with mice treated with PBS. To determine whether the PM-induced acceleration of thrombosis in the carotid artery was dependent on PM-induced activation of coagulation, we measured time to carotid occlusion in mice treated with enoxaparin (6 IU/animal 1 hour before the $\mathrm{FeCl}_{3}$ carotid artery injury). Treatment with enoxaparin prevented carotid artery occlusion (occlusion was not detected during 12 minutes of observation) both in PBS- and PM-treated mice, confirming that fibrin formation is required for carotid occlusion in this model. The PM-induced thrombocytosis that we observed might also have contributed to the shortening of the carotid artery occlusion time.

Exposure to PM increases the cell count and the levels of proinflammatory cytokines, particularly IL-6, in BAL fluid. We hypothesized that an inflammatory response to PM in the lung might be required for the systemic prothrombotic tendency observed in PM-treated mice. We performed cell count and differential analysis and measured the levels of proinflammatory cytokines in the BAL fluid 24 hours after treatment with either PBS or PM. In comparison with PBS-treated mice, animals exposed to PM had increased total cell counts, and the majority of the increase was attributable to an increase in macrophages, with a smaller increase in the number of neutrophils (Figure 4A). Compared with PBS-treated mice, PM-treated animals demonstrated a marked elevation in levels of IL-6 (16-fold) (Figure 4B) and modest increases in TNF- $\alpha$ and IFN- $\gamma$, while the levels of monocyte chemoattractant protein $1, \mathrm{IL}-12$, and the antiinflammatory cytokine IL-10 were not significantly altered (Figure 4B).

Alveolar macrophages are required for the PM-induced tendency toward thrombosis. To ascertain the contribution of alveolar macrophages to the PM-induced prothrombotic tendency, we depleted alveolar macrophages in mice with intratracheal liposomal clodronate 48 hours before exposure to $\mathrm{PM}_{10}$ or PBS. We used flow cytometry to measure the number of cells expressing the macrophage marker (anti-F4/80 antibody; Spherotech) in the BAL fluid 24 hours after the administration of clodronate. When compared with shamtreated (empty liposome-treated) animals, animals treated with clodronate had a reduction in the percentage of macrophages expressing F4/80 in the BAL fluid from $82 \%$ to $32 \%$. PM-induced changes in the bleeding time, PT, aPTT, platelet count, factor VIII activity (Figure 5A), and plasma TAT levels (Figure 5B) were not observed in mice depleted of alveolar macrophages with liposomal clodronate. In these animals, PM exposure also failed to shorten the time to cessation of blood flow following the application of $\mathrm{FeCl}_{3}$ to the carotid artery (Figure 5C).

IL-6 is required for the PM-induced tendency toward thrombosis. To determine whether the release of IL- 6 was required for the PM-induced prothrombotic tendency, we measured bleeding time, PT, aPTT, platelet count, factor VIII activity, and plasma TAT levels in $I L 6^{-/-}$mice 24 hours after exposure to PBS or PM. PM-induced changes in the bleeding time, PT, aPTT, platelet count, factor VIII activity (Figure 6A), and plasma TAT levels (Figure 6B) were not observed in $I L 6^{-/-}$mice. In these animals, PM exposure also failed to shorten the time to cessation of blood flow following the application of $\mathrm{FeCl}_{3}$ to the carotid artery (Figure 6C). Exposure to PM failed to cause a change in fibrinogen level (data not shown).

Alveolar macrophages are required for PM-induced increase in BAL IL-6. To determine the contribution of alveolar macrophages to PMinduced inflammatory changes in the lung, we measured BAL total and differential cell count and the levels of proinflammatory cytokines in mice treated with liposomal clodronate followed by PM or PBS. Compared with sham-treated mice, animals treated with liposomal clodronate had lower total number of cells and number of macrophages in response to PM (Figure 7A). However, we still observed a small increase in BAL fluid neutrophil count. The PMinduced increase in BAL fluid IL-6 level was attenuated in clodronate- 
A

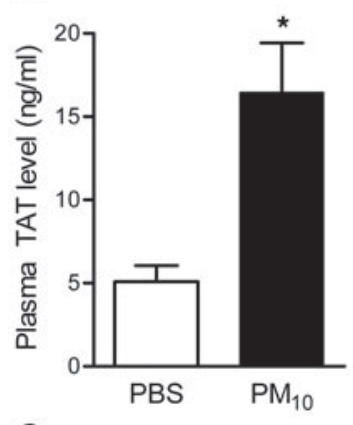

C

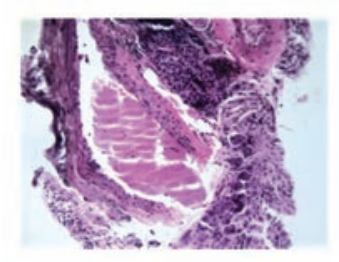

B
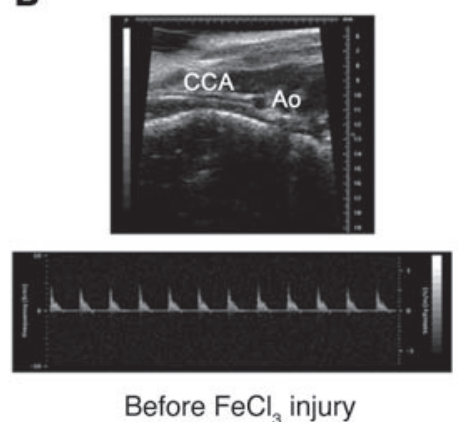

D

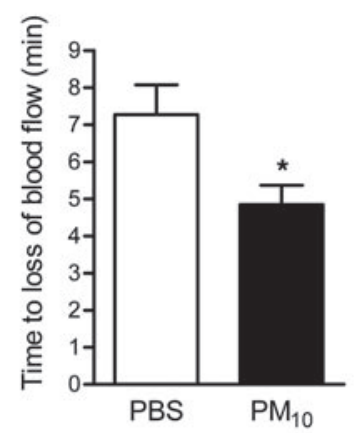

Figure 3

Effect of PM on thrombin generation and arterial thrombosis. (A) Plasma levels of TAT complexes in PM- and PBS-treated mice. (B-D) Time to occlusion of the carotid artery after the application of $\mathrm{FeCl}_{3}$. (B) Representative $2 \mathrm{D}$ ultrasound view of the common carotid artery (CCA) and aorta (Ao) and Doppler from CCA before and after application of $\mathrm{FeCl}_{3}$ (at the time of cessation of blood flow). Loss of blood flow detected via Doppler was also associated with dilation of CCA. (C) Representative histology of CCA after $\mathrm{FeCl}_{3}$ injury showing a large thrombus with almost complete occlusion of arterial lumen (original magnification, $\times 200$ ). (D) Time to loss of carotid blood flow in PM- or PBS-treated mice. ${ }^{*} P<0.05$ for comparison between PM- and PBS-treated mice, $n \geq 4$ in each treatment group. treated animals $(6.18 \pm 0.55$ and $40.05 \pm 17.24 \mathrm{pg} / \mathrm{ml}$ in clodronatetreated mice compared with $6.9 \pm 0.85$ and $164.25 \pm 53.63 \mathrm{pg} / \mathrm{ml}$ in wild-type mice after PBS and PM treatment, respectively) (Figure 7B). The levels of other proinflammatory cytokines after PM treatment in clodronate-treated mice were similar to sham-treated mice except for a modest elevation in the IL-10 level in liposomal clodronate-treated animals $(24.03 \pm 2.90$ in sham-treated mice versus $53.25 \pm 9.78 \mathrm{pg} / \mathrm{ml}$ in liposomal clodronate-treated mice) (Figure 7B).

We repeated these measurements in IL-6 knockout mice (IL6 $6^{-/-}$ mice). The PM-induced changes in cell count and differential in $I L 6^{-/-}$mice were similar to those in wild-type mice (Figure $7 \mathrm{C}$ ). Exposure to PM caused a small but significant increase in BAL IL-10 levels, as seen in clodronate-treated mice (Figure 7D).

To determine the effects of PM on systemic levels of cytokines, we measured plasma levels of proinflammatory cytokines in wildtype mice (control), wild-type mice treated with liposomal clodronate, and $I L 6^{--}$mice 24 hours after exposure to PM or saline. The pattern of elevation of systemic cytokine levels in response to PM was similar to that observed in BAL fluid but blunted (Figure 8).

\section{Discussion}

Ischemic cardiovascular events are the main contributor to excess mortality that is attributable to ambient PM $(1-3,6)$. Our results demonstrate that acute exposure of mice to moderate levels of ambient PM causes an enhanced tendency toward thrombosis sufficient to cause intravascular thrombin generation and accelerate arterial thrombosis after injury. The bleeding time is shortened and the platelet count is increased, the PT and partial thromboplastin time are decreased, and fibrinogen and factors II, VIII, and X are increased. Similar to the findings of other investigators, we found that exposure to PM increased the levels of inflammatory cells and cytokines in the BAL fluid. The largest increase was observed in the levels of IL-6, which has been linked to the activation of coagulation in other models and has been shown to be elevated in the serum of humans exposed to high levels of PM (9). Exposure of mice to PM was sufficient to induce the intravascular generation of TAT and to accelerate the time to carotid artery occlusion following $\mathrm{FeCl}_{3}$ induced injury, an effect inhibited by low-molecular-weight heparin. Consistent with the importance of IL- 6 in this response, mice lacking IL-6 did not develop an enhanced tendency toward thrombosis, generate TAT, or alter the time to carotid artery occlusion after injury. Depletion of alveolar macrophages by the intratracheal administration of liposomal clodronate prevented PM-induced IL-6 production and the enhanced tendency toward thrombosis. These findings demonstrate the importance of alveolar macrophages and IL-6 in the thrombotic response induced by PM.

Available data suggest that exposure to PM causes oxidant stress in inflammatory and epithelial cells in the lung. This results in lung epithelial dysfunction (15), increased susceptibility to lung injury, inflammation, and the release of inflammatory cytokines $(23,24)$, which may contribute to the mortality attributable to PM (17). Consistent with our results, other investigators have observed evidence for PM-induced alterations in coagulation in animals and humans. In accord with our findings in mice, Baccarelli et al. recently found a significant reduction in the PT in residents of a region in Italy exposed to higher levels of PM compared to those exposed to lower levels (19). In other cohorts, exposure to PM has been shown to increase the levels of fibrinogen (25) and von Willebrand factor (20) and to induce peripheral arterial thrombosis (8). Our finding that IL-6 generated in the lung is required for PMinduced thrombosis provides what we believe to be a novel mechanistic link between the PM-induced inflammatory response and the development of systemic thrombosis.

Among its many activities, IL-6 plays a pivotal role in hemostasis. IL-6 induces a prothrombotic state by increasing expression of tissue factor, fibrinogen, factor VIII, and von Willebrand factor, activating endothelial cells, increasing platelet production, stabilizing fibrin clots, and reducing the levels of inhibitors of hemostasis such as anti- 

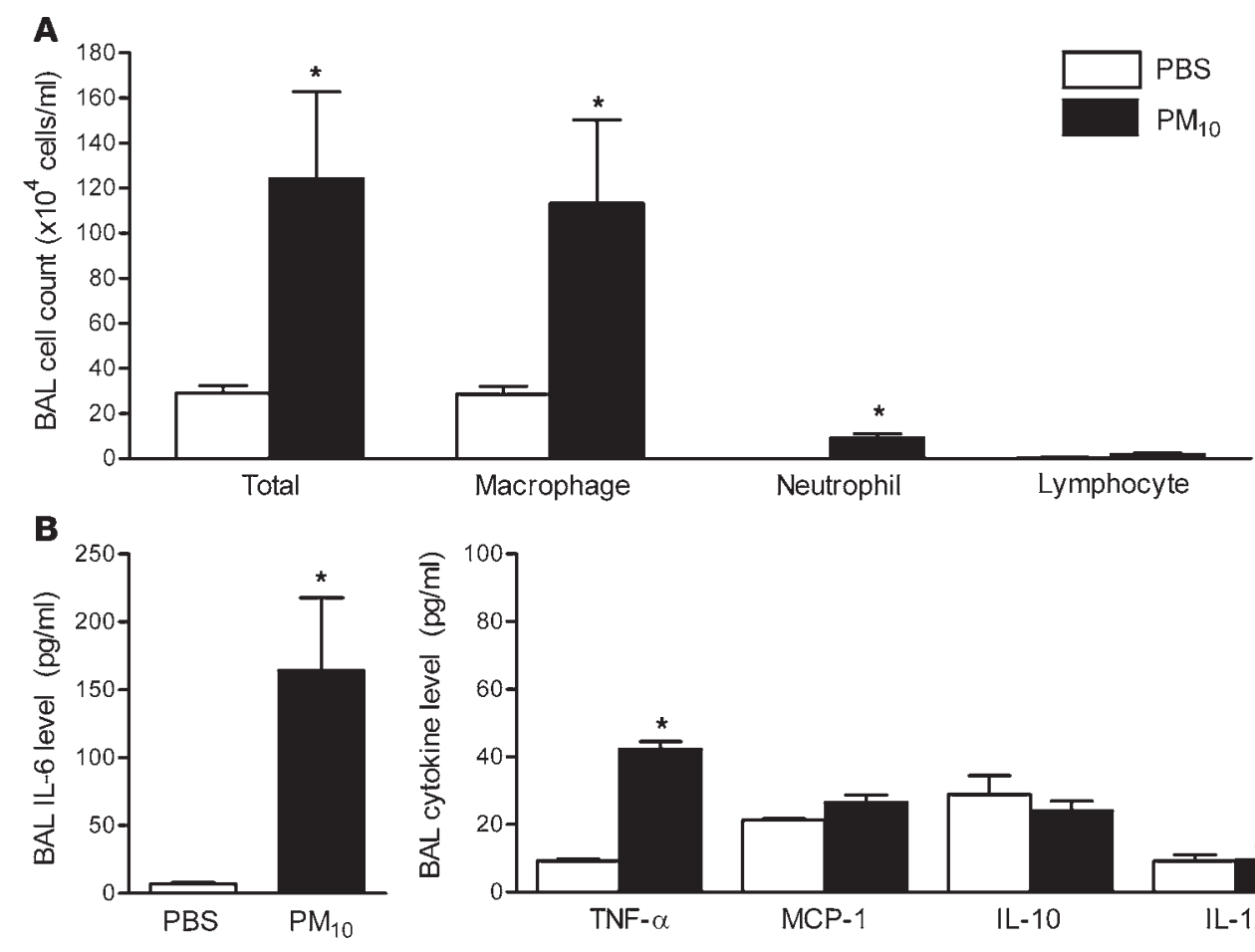

Neutrophil

ymphocyte

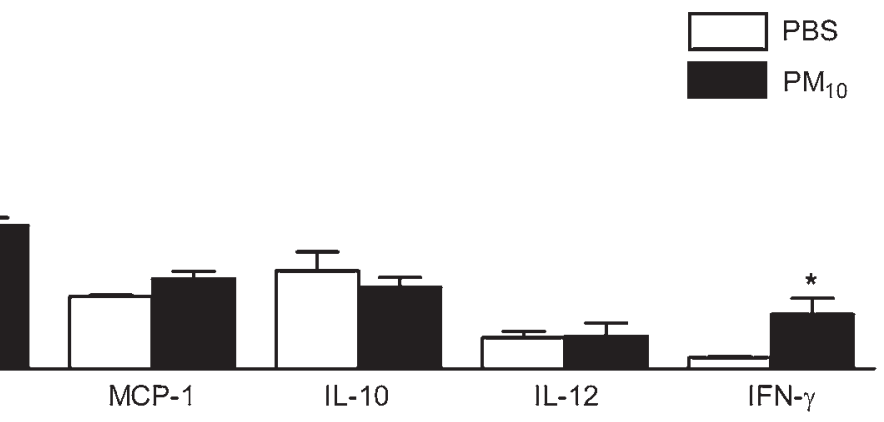

Figure 4

Alterations in BAL fluid total cell count and differential and the levels of inflammatory cytokines in wild-type mice treated with PM. (A) The total cell count and differential and (B) the levels of IL-6 and other inflammatory cytokines in the BAL fluid were measured 24 hours after the intratracheal instillation of $\mathrm{PM}_{10}$ or PBS. ${ }^{\star} P<0.05$ for comparison between $\mathrm{PM}$ - and PBS-treated mice, $n \geq 5$ in each treatment group.

thrombin and protein S (10, 26-28). IL-6 promotes coagulation and increases the generation of thrombin without affecting fibrinolysis (29). By increasing the transcription of factor VIII (30) and fibrinogen (31), IL-6 may increase the risk of both venous and arterial thrombosis, resulting in an increased risk of coronary artery disease (32) and venous thromboembolism $(33,34)$. By contrast, transcription of the factor VIII gene is not affected by other cytokines such as TNF- $\alpha$, IL-1, and IL-2 (30). In our model, the PM-induced reduction in the PT was attributable to an increase in factor II and factor X without a change in factor $\mathrm{V}$ or factor VII. The modest increases in the levels of factor VIII and fibrinogen suggest a mild acute phase response. This may in part account for the thrombocytosis observed in response to PM. Alternatively, the thrombocytosis may be attributable to a direct effect of IL-6 through the sequential activation of gp130 and STAT3 in bone marrow progenitor cells (35). Independent strategies that attenuated IL- 6 production, including IL- 6 knockout mice, and depletion of alveolar macrophages with liposomal clodronate prevented the PM-induced prothrombotic tendency.

The association between IL- 6 and ischemic heart disease is well established and is substantially attributable to the effects of IL- 6 on hemostasis (reviewed in refs. 11, 12). IL-6 levels correlate well with risk factors for cardiovascular disease such as age, fibrinogen, white blood cell count, and blood viscosity (36). IL-6 induces acute phase reactant release from hepatocytes (37), and one of these acute phase reactants, C-reactive protein, is a useful surrogate marker of IL-6 (38). Increased IL- 6 and C-reactive protein levels are independently associated with increased relative risk for mortality from cardiovascular disease (13). In support of the importance of IL-6 in PM-induced cardiovascular events in humans, Riediker et al. observed increased
C-reactive protein levels in highway patrol officers after exposure to traffic-related PM (20). In 57 men with coronary artery disease, Ruckerl and colleagues reported a similar correlation between PM exposure and the serum level of C-reactive protein (9).

Treatment with PM induced a dramatic increase in the levels of IL-6 and more modest increases in other proinflammatory cytokines in the BAL fluid including TNF- $\alpha$ and IFN- $\gamma$. The most dramatic difference in cytokines observed between wild-type mice and mice treated with liposomal clodronate or $\mathrm{IL6}^{-/-}$mice was the failure to induce IL-6 in response to PM. However, both of these latter groups exhibited small but significant increases in the levels of IL-10 in response to PM and neither of these groups of animals exhibited the modest increase in IFN- $\gamma$ observed in control animals after treatment with PM. Treatment with liposomal clodronate resulted in a significant reduction in the level of IL- 6 but not TNF- $\alpha$ in the BAL fluid from PM-treated animals. This suggests that while macrophages are responsible for the bulk of the IL- 6 production in response to PM, other cells in the lung, for example airway epithelial cells or neutrophils, are responsible for the PM-induced production of TNF- $\alpha$. These results are consistent with our observation that treatment with liposomal clodronate failed to abrogate the PM-induced increase in neutrophils in the lung. Collectively these results suggest that the generation of IL- 6 by lung macrophages is required for the prothrombotic tendency induced by PM exposure, but they leave open the possibility that other factors, for example the activation of neutrophils, other inflammatory cells, or alterations in the levels of other cytokines, might be required downstream of or in parallel with IL-6.

Our results implicating lung macrophages in the response to PM are consistent with reports from other investigators. For 
A

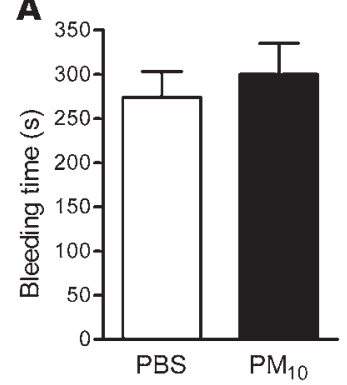

B

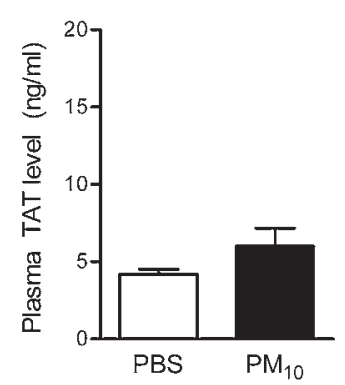

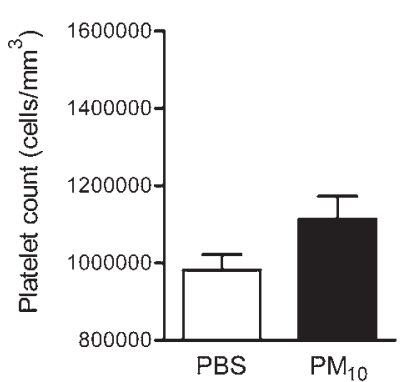
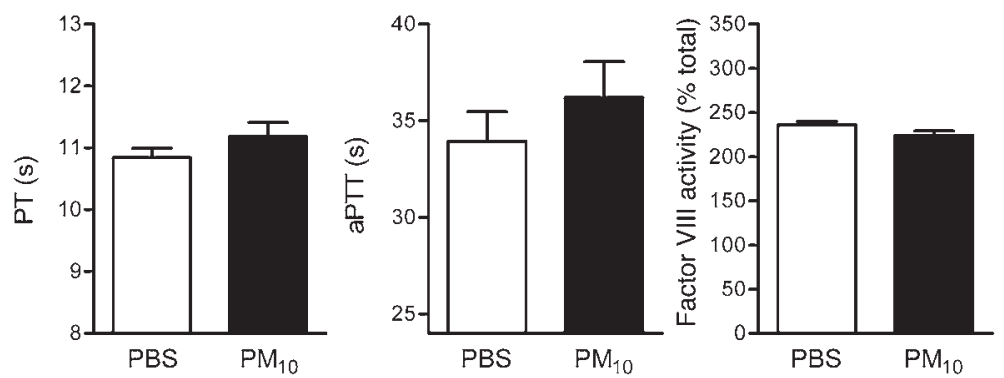

C

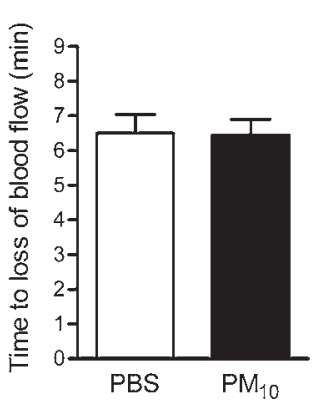

Figure 5

Effect of alveolar macrophage depletion on PM-induced alterations in hemostatic indices, thrombin generation, and arterial thrombosis. (A) Measurements of hemostasis, including bleeding time, platelet count, PT, aPTT, and factor VIII activity. (B) TAT complex plasma levels. (C) Time to occlusion of the carotid artery after the application of $\mathrm{FeCl}_{3}$ in $\mathrm{PM}$ - and PBS-treated wild-type mice depleted of alveolar macrophages using liposomal clodronate. $n \geq 5$ in each treatment group.

example, Nemmar and colleagues found that the intratracheal administration of clodronate to hamsters prevented the inflammatory response to silica in the lung and the subsequent development of peripheral thrombosis (39). Furthermore, postmortem examination of human lungs from areas where the average
PM levels were high revealed that particulates accumulated in alveolar macrophages (40).

The increase in cellularity in the BAL fluid was similar in wildtype animals and $\mathrm{ILG}^{-/-}$animals; the majority of the increase was attributable to an increase in macrophages, with a smaller increase
A

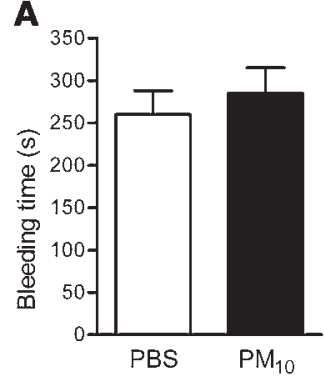

B

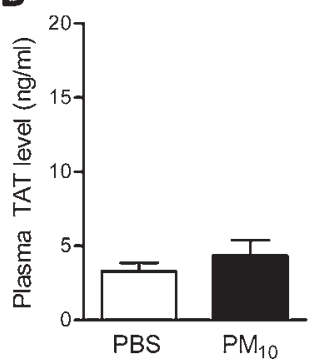

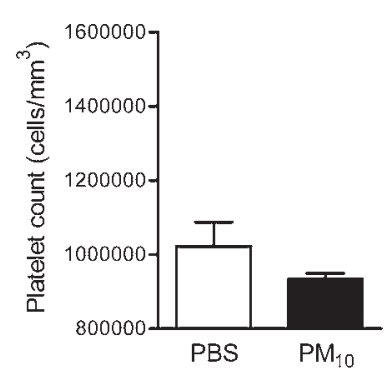

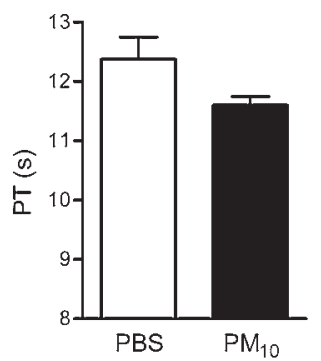

C

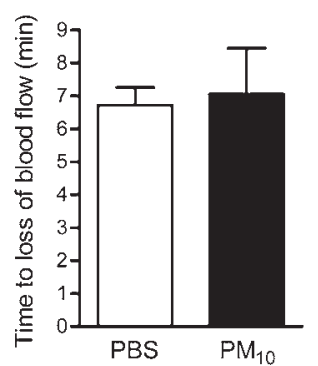

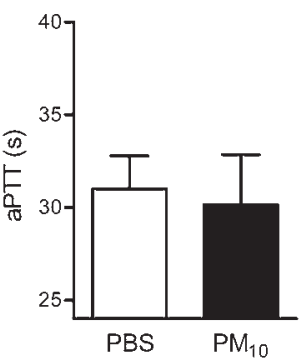

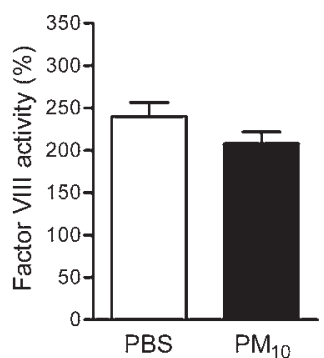

\section{Figure 6}

Effect of PM exposure on hemostatic indices, thrombin generation, and arterial thrombosis in IL6 ${ }^{-/-}$mice. (A) Measurements of hemostasis, including bleeding time, platelet count, PT, aPTT, and factor VIII activity. (B) TAT complex plasma levels. (C) Time to occlusion of the carotid artery after the application of $\mathrm{FeCl}_{3}$ in $\mathrm{PM}$ - and PBS-treated IL6-/- mice. $n \geq 5$ in each treatment group. 

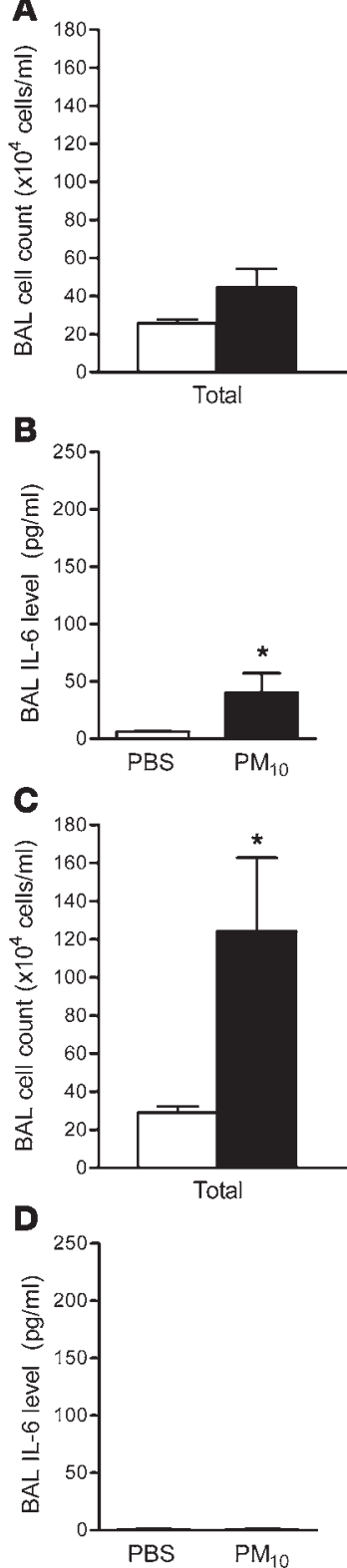
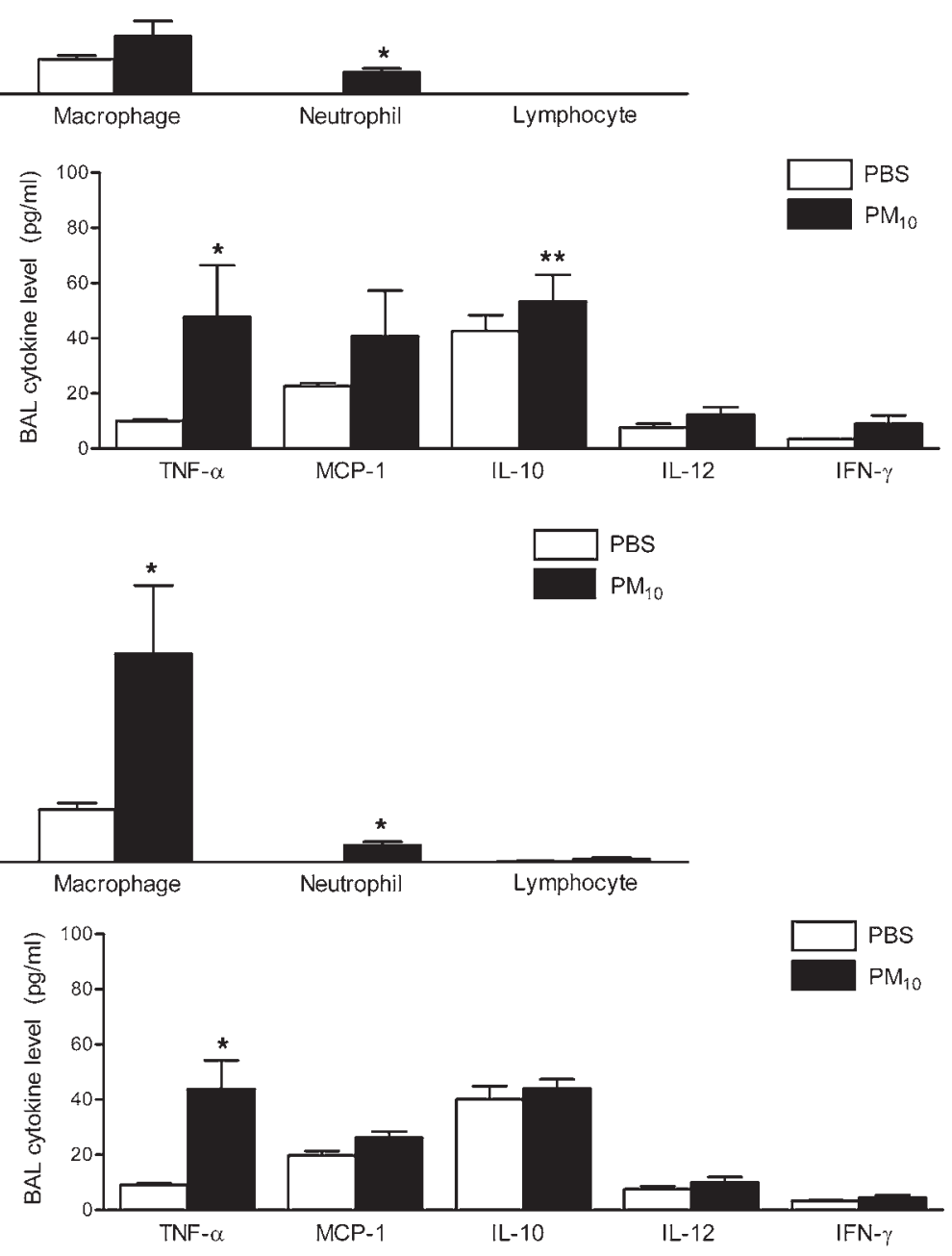

in the number of neutrophils. These results strongly suggest that IL-6 exerts its effects downstream of the development of lung inflammation. The central importance of lung inflammation in the response to PM is further supported by our observation that the increase in proinflammatory cytokines in the BAL fluid was substantially larger than that observed in the plasma. This is the same pattern that has been reported in other forms of injury that primarily involve the lung (41).

The dose of PM we used in our in vivo model is comparable to the dose used in previous animal models of PM exposure (8). These investigators reported that the intratracheal instillation of $35 \mu \mathrm{g}$ of PM to hamsters (8), whose minute ventilation is similar to mice, is sufficient to induce intravascular thrombosis (42). More importantly, the dose used is close to the range of $\mathrm{PM}_{10}$ to which humans might be exposed. In 2002, the United States Environmental Protection Agency reported a range of maximal city $\mathrm{PM}_{10}$ concentrations between 26 and $534 \mu \mathrm{g} / \mathrm{m}^{3}$ (17). Many large cities

\section{Figure 7}

Effect of PM exposure on BAL fluid total cell count and differential and the levels of inflammatory cytokines in $I L 6^{-/-}$mice and wild-type mice depleted of alveolar macrophages. The total cell count and differential (A) and the levels of IL-6 and other inflammatory cytokines (B) in the BAL fluid were measured 24 hours after the intratracheal instillation of $\mathrm{PM}_{10}$ or PBS in wild-type mice treated with liposomal clodronate. (C and D) The same measurements were performed in $I L 6^{-/-}$mice. ${ }^{*} P<0.05$ for comparison between $\mathrm{PM}$ and PBS treated mice; ${ }^{* *} P<0.05$ for comparison between shamtreated and liposomal clodronate-treated wild-type mice exposed to PM and between wild-type mice and $I L 6^{-/-}$mice exposed to PM. $n \geq 5$ in each treatment group. 

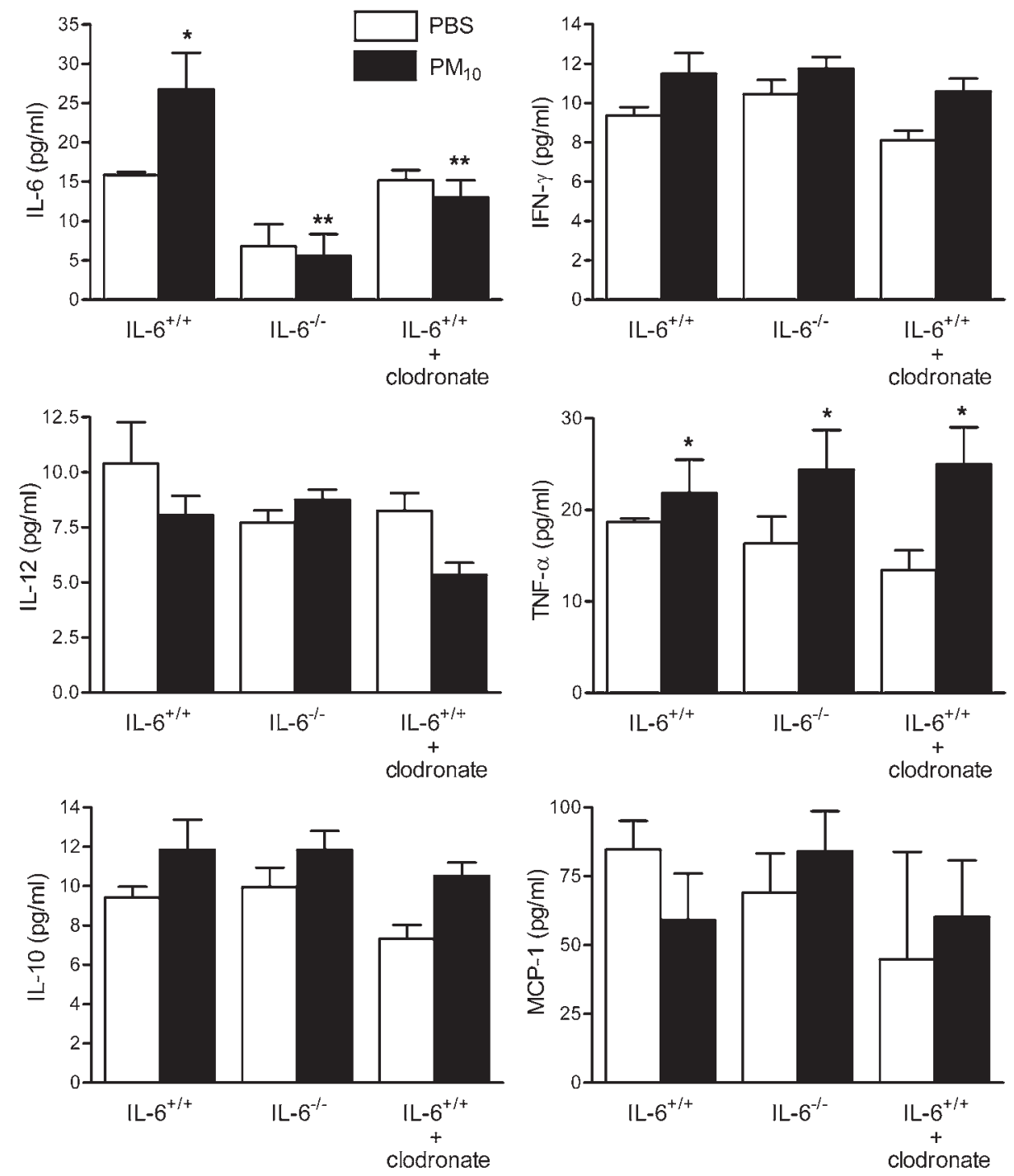

Figure 8

Effect of PM exposure on systemic cytokines. Systemic levels of IL-6 and other inflammatory cytokines were measured 24 hours after the intratracheal instillation of $\mathrm{PM}_{10}$ or PBS in wild-type mice, IL6 ${ }^{-1-}$ mice, and wild-type mice with alveolar macrophage depletion (treated with liposomal clodronate 48 hours before treatment with PM or PBS). ${ }^{*} P<0.05$ for comparison between PM- and PBS-treated mice, $n \geq 5$ in each treatment group.

\section{Methods}

$P M$. The PM was collected by baghouse from ambient air in Düsseldorf, Germany. The particle sample was aerosolized from a turntable into a small-scale powder disperser utilizing a high airflow to break up aggregates in the Venturi throat. The characteristics of the PM have been previously reported (16). We used $\mathrm{PM}_{10}$ in our experiments.

Animals and intratracheal administration of PM. The protocol for the use of mice was approved by the Animal Care and Use Committee at Northwestern University. We used 6- to 8-week-old (20-25 g) male C57BL/6 mice (IL6 ${ }^{+/+}$mice) and mice with targeted deletion of IL-6 (IL6-/-; B6.129S2-

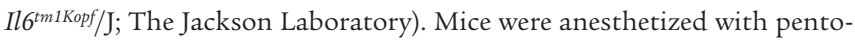
barbital ( $50 \mathrm{mg} / \mathrm{kg}$ intraperitoneally) and intubated orally with a 20-gauge angiocath (45). We instilled either $\mathrm{PM}_{10}$ suspended in $50 \mu \mathrm{l}$ of sterile PBS or $50 \mu \mathrm{l}$ of sterile PBS (control) in 2 equal aliquots, 3 minutes apart. PM was vortexed prior to instillation. After each aliquot the mice were placed in the right and then the left lateral decubitus position for $10-15$ seconds.

Coagulation studies and bleeding time. Bleeding time was measured by cutting the tail vein as previously described (46). For coagulation studies, venous blood was collected via direct right atrial puncture. We collected the blood for complete blood count and coagulation parameters, which were analyzed with STA Compact Coagulation Analyzer (Diagnostica Stago). For measurement of TAT complex levels, we used Enzygnost TAT Micro kit (Dade Behring Inc.). Factor VIII activity was measured using a chromogenic substrate assay (Dade Behring Inc.), which depends on the activation of factor $\mathrm{X}$ by factor VIIIa, factor IXa, phospholipids, and calcium ions.

$B A L$ analysis. BAL was performed through a 20 -gauge angiocath ligated into the trachea. A 1.0-ml aliquot of PBS was instilled into mouse lungs and then slowly aspirated 3 times. A $200 \mu$ l aliquot of the BAL fluid was placed in a cytospin and centrifuged at $100 \mathrm{~g}$ for 5 minutes. The supernatant was used to measure IL-6 and cytokine/chemokine levels. We used BD Cytometric Bead Array (BD Biosciences) to measure systemic and BAL levels of cytokines/chemokines. Samples were analyzed in triplicate using the Mouse Inflammation Kit (BD Biosciences), which detects IL-6, -10 , and -12 , monocyte chemoattractant protein 1 , IFN- $\gamma$, and TNF- $\alpha$, according to the instructions provided.

Alveolar macrophage depletion. Liposomal encapsulation of clodronate (dichloromethylene diphosphonate) was performed as previously described (47). Clodronate $(120 \mathrm{mg})$ was instilled into mouse lungs following endotracheal intubation 48 hours before instillation of PM or saline. Depletion of macrophages was confirmed by assessment of BAL fluid.

Carotid artery thrombosis and ultrasound (22). After adequate anesthesia was achieved, the left carotid artery was dissected and a $0.5 \times 1.0-\mathrm{mm}$ strip of Whatman No. 1 filter paper soaked in $10 \% \mathrm{FeCl}_{3}$ solution was applied to the surface of the adventitia for 30 seconds. This led to a 2- to 3-mmlong carotid thrombus. Both aorta and left common carotid artery were visualized, and carotid blood flow was documented before and after $\mathrm{FeCl}_{3}$ application and monitored continuously using a cardiac ultrasound highresolution imaging/Doppler from parasternal long axis views. All imaging was performed using the cardiovascular scanhead RMV 707B and Vevo 770 echocardiography equipment, which offers high resolution down to $30 \mu \mathrm{m}$ (Visualsonics Inc.).

Flow-cytometric analysis of platelets and alveolar macrophages. Mouse platelet activation was analyzed by flow cytometry using a PE-conjugated antibody against the pan-platelet antigen $\alpha_{\text {IIb }} \beta_{3}$ a and a FITC-conjugated antibody against the platelet activation marker P selectin (CD62P) (both antibodies 
from Emfret Analytics) in the absence or presence of ADP as previously described (21). Briefly, blood was obtained from the retroorbital venous plexus using a heparinized glass syringe and diluted (1:25) in Tyrode's buffer with $\mathrm{CaCl}_{2}$, then incubated with either a control antibody or FITClabeled anti-CD62P antibody with or without $\operatorname{ADP}(25 \mu \mathrm{M})$ and analyzed using FACS gating by cell size and PE/FITC labeling.

Macrophages in the BAL fluid were measured as previously described (48). Briefly, freshly obtained BAL fluid was centrifuged $(600 \mathrm{~g})$ and the resulting pellet was washed twice in ice-cold PBS then resuspended in PBS with 5\% BSA containing a FITC-labeled antibody against the macrophage antigen $\mathrm{F} 4 / 80$ (Spherotec), incubated with rotation for $30 \mathrm{~min}$ utes, washed in PBS, and resuspended in PBS with $0.5 \%$ PFA containing DAPI (Invitrogen) and then analyzed by flow cytometry. The percentage of macrophages was expressed as the percentage of DAPI-positive cells that were also FITC positive.

Statistics. Results are expressed as mean \pm SD. The observed results were similar in independently performed iterations of the experiment. Data were analyzed using 1-way ANOVA. When ANOVA indicated that a significant difference was present, we explored individual differences with the 2-tailed
Student's $t$ test using Bonferroni correction for multiple comparisons (Prism 4; GraphPad Software). Statistical significance was defined as $P<0.05$.

\section{Acknowledgments}

This study was supported by grants from the American Lung Association and American Lung Association of Metropolitan Chicago (to G.M. Mutlu), and NIH grants HL059956 (to D.A. Dean), ES013995 (to G.R.S. Budinger), HL071643 (to N.S. Chandel, D.A. Dean, G.R.S. Budinger, and J.I. Sznajder), and ES015024 (National Institute of Environmental Health Sciences [NIEHS] Outstanding New Environmental Scientist [ONES] award [to G.M. Mutlu]).

Received for publication October 13, 2006, and accepted in revised form July 6, 2007.

Address correspondence to: Gökhan M. Mutlu, Northwestern University Feinberg School of Medicine, 240 E. Huron Street, McGaw M-300, Chicago, Illinois 60611, USA. Phone: (312) 908-8163; Fax: (312) 908-4650; E-mail: g-mutlu@northwestern.edu.
1. Peters, A., Dockery, D.W., Muller,J.E., and Mittleman, M.A. 2001. Increased particulate air pollution and the triggering of myocardial infarction. Circulation. 103:2810-2815.

2. D'Ippoliti, D., et al. 2003. Air pollution and myocardial infarction in Rome: a case-crossover analysis. Epidemiology. 14:528-535.

3. Peters, A., et al. 2004. Exposure to traffic and the onset of myocardial infarction. N. Engl. J. Med. 351:1721-1730.

4. Rich, D.Q., et al. 2005. Association of short-term ambient air pollution concentrations and ventricular arrhythmias. Am. J. Epidemiol. 161:1123-1132.

5. Wellenius, G.A., Bateson, T.F., Mittleman, M.A., and Schwartz, J. 2005. Particulate air pollution and the rate of hospitalization for congestive heart failure among Medicare beneficiaries in Pittsburgh, Pennsylvania. Am. J. Epidemiol. 161:1030-1036.

6. Wellenius, G.A., Schwartz, J., and Mittleman, M.A. 2005. Air pollution and hospital admissions for ischemic and hemorrhagic stroke among Medicare beneficiaries. Stroke. 36:2549-2553.

7. Nemmar, A., Nemery, B., Hoet, P.H., Vermylen, J., and Hoylaerts, M.F. 2003. Pulmonary inflammation and thrombogenicity caused by diesel particles in hamsters: role of histamine. Am. J. Respir. Crit. Care Med. 168:1366-1372.

8. Nemmar, A., et al. 2003. Diesel exhaust particles in lung acutely enhance experimental peripheral thrombosis. Circulation. 107:1202-1208.

9. Ruckerl, R., et al. 2006. Air pollution and markers of inflammation and coagulation in patients with coronary heart disease. Am. J. Respir. Crit. Care Med. 173:432-441

10. Kerr, R., Stirling, D., and Ludlam, C.A. 2001. Interleukin 6 and haemostasis. Br. J. Haematol. 115:3-12.

11. Tracy, R.P. 1999. Epidemiological evidence for inflammation in cardiovascular disease. Thromb. Haemost. 82:826-831.

12. Yudkin, J.S., Stehouwer, C.D., Emeis, J.J., and Coppack, S.W. 1999. C-reactive protein in healthy subjects: associations with obesity, insulin resistance, and endothelial dysfunction: a potential role for cytokines originating from adipose tissue? Arterioscler. Thromb. Vasc. Biol. 19:972-978.

13. Harris, T.B., et al. 1999. Associations of elevated interleukin-6 and C-reactive protein levels with mortality in the elderly. Am. J. Med. 106:506-512.

14. Yeh, E.T. 2004. CRP as a mediator of disease. Circulation. 109:II11-II14.

15. Mutlu, G.M., et al. 2006. Airborne particulate mat- ter inhibits alveolar fluid reabsorption in mice via oxidant generation. Am. J. Respir. Cell Mol. Biol. 34:670-676.

16. Upadhyay, D., Panduri, V., Ghio, A., and Kamp, D.W. 2003. Particulate matter induces alveolar epithelial cell DNA damage and apoptosis: role of free radicals and the mitochondria. Am. J. Respir. Cell Mol. Biol. 29:180-187.

17. Brook, R.D., et al. 2004. Air pollution and cardiovascular disease: a statement for healthcare professionals from the Expert Panel on Population and Prevention Science of the American Heart Association. Circulation. 109:2655-2671.

18. Ghio, A.J., Hall, A., Bassett, M.A., Cascio, W.E., and Devlin, R.B. 2003. Exposure to concentrated ambient air particles alters hematologic indices in humans. Inhal. Toxicol. 15:1465-1478.

19. Baccarelli, A., et al. 2007. Effects of exposure to air pollution on blood coagulation. J. Thromb. Haemost. 5:252-260.

20. Riediker, M., et al. 2004. Particulate matter exposure in cars is associated with cardiovascular effects in healthy young men. Am. J. Respir. Crit. Care Med. 169:934-940.

21. Nieswandt, B., Schulte, V., and Bergmeier, W. 2004. Flow-cytometric analysis of mouse platelet function. Methods Mol. Biol. 272:255-268.

22. Konstantinides, S., Schafer, K., Thinnes, T., and Loskutoff, D.J. 2001. Plasminogen activator inhibitor- 1 and its cofactor vitronectin stabilize arterial thrombi after vascular injury in mice. Circulation. 103:576-583.

23. Nel, A. 2005. Atmosphere. Air pollution-related illness: effects of particles. Science. 308:804-806.

24. Li, N., et al. 2004. Nrf2 is a key transcription factor that regulates antioxidant defense in macrophages and epithelial cells: protecting against the proinflammatory and oxidizing effects of diesel exhaust chemicals. J. Immunol. 173:3467-3481.

25. Ghio, A.J., Kim, C., and Devlin, R.B. 2000. Concentrated ambient air particles induce mild pulmonary inflammation in healthy human volunteers. Am. J. Respir. Crit. Care Med. 162:981-988.

26. Burstein, S.A., et al. 1996. Cytokine-induced alteration of platelet and hemostatic function. Stem Cells. 14(Suppl. 1):154-162.

27. Neumann, F.J., et al. 1997. Effect of human recombinant interleukin- 6 and interleukin- 8 on monocyte procoagulant activity. Arterioscler. Thromb. Vasc. Biol. 17:3399-3405.

28. Niessen, R.W., et al. 1997. Antithrombin acts as a negative acute phase protein as established with studies on HepG2 cells and in baboons. Thromb. Haemost. 78:1088-1092.

29. Stouthard, J.M., et al. 1996. Interleukin-6 stimulates coagulation, not fibrinolysis, in humans. Thromb. Haemost. 76:738-742.

30. Stirling, D., Hannant, W.A., and Ludlam, C.A. 1998. Transcriptional activation of the factor VIII gene in liver cell lines by interleukin-6. Thromb. Haemost. 79:74-78.

31. Amrani, D.L. 1990. Regulation of fibrinogen biosynthesis: glucocorticoid and interleukin- 6 control. Blood Coagul. Fibrinolysis. 1:443-446.

32. Kannel, W.B., Wolf, P.A., Castelli, W.P., and D'Agostino, R.B. 1987. Fibrinogen and risk of cardiovascular disease. The Framingham Study. JAMA. 258:1183-1186.

33. Kraaijenhagen, R.A., et al. 2000. High plasma concentration of factor VIIIc is a major risk factor for venous thromboembolism. Thromb. Haemost. 83:5-9.

34. Kyrle, P.A., et al. 2000. High plasma levels of factor VIII and the risk of recurrent venous thromboembolism. N. Engl. J. Med. 343:457-462.

35. Jenkins, B.J., et al. 2007. Pathologic consequences of STAT3 hyperactivation by IL- 6 and IL-11 during hematopoiesis and lymphopoiesis. Blood. 109:2380-2388.

36. Woodward, M., Rumley, A., Tunstall-Pedoe, H., and Lowe, G.D. 1999. Associations of blood rheology and interleukin- 6 with cardiovascular risk factors and prevalent cardiovascular disease. Br. J. Haematol. 104:246-257.

37. Gauldie, J., Northemann, W., and Fey, G.H. 1990. IL-6 functions as an exocrine hormone in inflammation. Hepatocytes undergoing acute phase responses require exogenous IL-6. J. Immunol. 144:3804-3808.

38. Heinrich, P.C., Castell, J.V., and Andus, T. 1990. Interleukin- 6 and the acute phase response. Biochem. J. 265:621-636.

39. Nemmar, A., Nemery, B., Hoet, P.H., Van Rooijen, N., and Hoylaerts, M.F. 2005. Silica particles enhance peripheral thrombosis: key role of lung macrophage-neutrophil cross-talk. Am. J. Respir. Crit. Care Med. 171:872-879.

40. Brauer, M., et al. 2001. Air pollution and retained particles in the lung. Environ. Health Perspect. 109:1039-1043.

41. Imai, Y., et al. 2003. Injurious mechanical ventilation and end-organ epithelial cell apoptosis and organ dysfunction in an experimental model of acute respiratory distress syndrome. JAMA. 289:2104-2112. 
42. Schlenker, E.H. 1984. An evaluation of ventilation in dystrophic Syrian hamsters. J. Appl. Physiol. 56:914-921.

43. [Anonymous]. 1994. Air pollution in the world's megacities: a report from the UN Environment Programme and WHO Environment. United Nations. New York, New York, USA. WHO. Geneva, Switzerland. Environment. 36:35-37.

44. de Hennezel, L., et al. 2001. Plethysmography for the assessment of pneumococcal pneumonia and passive immunotherapy in a mouse model. Eur. Respir. J. 17:94-99.

45. Mutlu, G.M., et al. 2004. Upregulation of alveolar epithelial active $\mathrm{Na}+$ transport is dependent on beta2-adrenergic receptor signaling. Circ. Res. 94:1091-1100.

46. Gailani, D., Lasky, N.M., and Broze, G.J., Jr. 1997. A murine model of factor XI deficiency. Blood Coagul.
Fibrinolysis. 8:134-144.

47. Everhart, M.B., et al. 2005. Intratracheal administration of liposomal clodronate accelerates alveolar macrophage reconstitution following fetal liver transplantation. J. Leukoc. Biol. 77:173-180.

48. Garn, H., et al. 2006. Phenotypical and functional characterization of alveolar macrophage subpopulations in the lungs of $\mathrm{NO} 2$-exposed rats. Respir. Res. 7:4. 